\title{
Long-term ex vivo monitoring of in vivo microRNA activity in liver using a secreted luciferase sensor
}

\author{
WANG Gang $^{1 \dagger}$, DONG XiaoYan ${ }^{1,2,3 \dagger}$, HU JianYang ${ }^{1,4}$, TIAN WenHong ${ }^{1}$, YUCHI Jie ${ }^{1}$, \\ WANG Yue ${ }^{1 *} \&$ WU XiaoBing ${ }^{1 *}$ \\ ${ }^{1}$ State Key Laboratory of Molecular Virology and Genetic Engineering, National Institute for Viral Disease Control and Prevention, \\ Chinese Center for Disease Control and Prevention, Beijing 100052, China; \\ ${ }^{2}$ State Key Laboratory of Genetic Engineering, Institute of Genetics, School of Life Sciences, \\ Fudan University, Shanghai 200433, China; \\ ${ }^{3}$ Beijing FivePlus Molecular Medicine Institute, Beijing 100176, China; \\ ${ }^{4}$ Department of Hepatobiliary Surgery, Affiliated Hospital of Ningxia Medical University, Yinchuan 750004, China
}

Received July 27, 2010; accepted April 18, 2011

\begin{abstract}
Technology for monitoring in vivo microRNA (miRNA) activity is extremely important for elucidating miRNA biology. However, in vivo studies of miRNA have been hampered by the lack of a convenient approach to reliably reflect real-time functional changes in miRNAs. Sensors for miRNA were developed by adding miRNA target sequences to the 3'-untranslated region of Gaussia princeps luciferase (Gluc) mRNA. These sensors were then evaluated in vitro and in vivo by measuring Gluc activity in cell supernatants and in peripheral blood. Sensors driven by the CMV promoter were effective for monitoring miR-122 in living cells, but not for the long-term monitoring of miR-122 or miR-142 in mouse liver because of CMV-promoter silencing. Replacing the CMV promoter with a CAG promoter rendered these sensors effective for the long-term monitoring of relevant liver miRNA activities. We subsequently used the CAG-promoter-based sensor for the long-term monitoring of endogenous liver miR-122, miR142 and miR-34a activities, as well as for exogenous miR-34a activity. Our study demonstrates that real-time in vivo activities of miRNAs can be continuously and conveniently detected in mouse liver using the sensors that we have developed.
\end{abstract}

microRNA sensor, secreted Gaussia luciferase, ex vivo monitoring

Citation: Wang G, Dong X Y, Hu J Y, et al. Long-term ex vivo monitoring of in vivo microRNA activity in liver using a secreted luciferase sensor. Sci China Life Sci, 2011, 54: 418-425, doi: 10.1007/s11427-011-4171-0

MicroRNAs (miRNAs) are a class of endogenous, small, functional RNAs of 19-23 nt. Mature miRNAs bind to complementary sequences in the $3^{\prime}$-untranslated regions (UTRs) of target mRNAs and cause either translational repression or mRNA degradation, and hence regulate protein expression [1-4].

Current approaches for miRNA detection are mainly based on Northern blotting, reverse transcription quantita-

†Contributed equally to this work

*Corresponding author (email: euy-tokyo@umin.ac.jp; wuxb0168@vip.sina.com) tive PCR (RT-qPCR) and microarray analysis [5-7]. While these methods have been widely used, they are both time-consuming and laborious. Furthermore, they do not reflect the real-time function of a given miRNA in living cells. A few miRNA "sensors", constructed by inserting a given miRNA target sequence into the 3 '-UTR of reporter genes, including lacZ [8], GFP [9] and luciferase [10-12], have been reported. Using these sensors, miRNA activity can be inferred by measuring the inhibition of reporter gene expression. Although these sensors may reveal the spatiotemporal expression patterns of miRNAs in tissues and may 
advance our understanding of miRNA functions, their usefulness in living organisms has not been fully evaluated. Furthermore, these sensors were not developed for the purpose of continuous and convenient ex vivo monitoring of in vivo miRNAs. Thus, miRNA sensors can be improved.

Recently, a secreted luciferase, Gluc, from the marine copepod Gaussia princeps has been used as a sensitive and quantitative tool for the in vivo non-invasive monitoring of transduced tumor cells inoculated in mice [13-15]. Tumor expansion can be monitored easily by measuring Gluc expression in blood. Thus, Gluc is an ideal candidate for improving miRNA sensors. In the present study, we generated a series of miRNA sensors by inserting miRNA target sequences into the 3'-UTR of the Gluc gene, and evaluated the suitability of different promoters for use in the mouse liver. We demonstrate that a CAG/Gluc-miRT sensor can be used to continuously and conveniently monitor miRNAs in mouse liver.

\section{Materials and methods}

\subsection{Sensor construction}

All sensors generated contained three major elements: a CMV or CAG promoter (the latter being a composite promoter consisting of the CMV IE enhancer, the chicken $\beta$-actin promoter, and a rabbit $\beta$-globin polyadenylation signal); a Gluc reporter gene derived from pGluc-Basic (New England Biolabs, Ipswich, USA); and four repeats of miR-122, miR-142 or miR-34a target sequences in the 3'-UTR of the Gluc gene. These elements were cloned into an adeno-associated virus serotype 2 (AAV2) expression vector with inverted terminal repeat (ITR) sequences flanking the transgene. We named the resulting sensors $\mathrm{CMV} /$ Gluc-miR-122T and CAG/Gluc-miR-122T; CMV/Gluc-miR142T and CAG/Gluc-miR-142T; and CMV/Gluc-miR-34aT and CAG/Gluc-miR-34aT (Figure 1A). The following oligonucleotides were used to generate the target sites: miR-122, 5'-AATTCCAAACACCATTGTCACACTCCAAGACCAAACACCATTGTCACACTCCAAGACCAAACACCATTGTCACACTCCAAGACCAAACACCATTGTCACACTCCAA-3' and 5'-GATCTTGGAGTGTGACAATGGTGTTTGGTCTTGGAGTGTGACAATGGTGTTTGGTCTTGGAGTGTGACAATGGTGTTTGGTCTTGGAGTGTGACAATGGTGTTTGG-3'; miR-142, 5'-AATTC ATAAAGTAGGAAACACTACAAGACTCCATAAAGTAGGAAACACTACAAGACTCCATAAAGTAGGAAACACTACAAGACTCCATAAAGTAGGAAACACTACAA$3^{\prime}$ and 5'-GATCTTGTAGTGTTTCCTACTTTATGGAGTCTTGTAGTGTTTCCTACTTTATGGAGTCTTGTAGTGTTTCCTACTTTATGGAGTCTTGTAGTGTTTCCTACTTTATGGAG-3'; and miR-34a, 5'-AATTCACAACCAGCTAAGACACTGCCAAGACACAACCAGCTAAGACACTGCCAAGACACAACCAGCTAAGACACTGCCAAGAC-
ACAACCAGCTAAGACACTGCCAA-3' and 5'-GATCTTGGCAGTGTCTTAGCTGGTTGTGTCTTGGCAGTGTCTTAGCTGGTTGTGTCTTGGCAGTGTCTTAGCTGGTTGTGTCTTGGCAGTGTCTTAGCTGGTTGTG-3'. Gluc expression plasmids under the control of the CMV or CAG promoter without miRNA binding sites were also constructed as controls and were named CMV/Gluc or CAG/Gluc. A firefly luciferase (Fluc) expression plasmid under the control of the CMV promoter without miRNA binding sites was also constructed as an internal control for the Gluc-based sensor and was named CMV/Fluc. The pre-miR-122 and pre-miR-34a genes were amplified from genomic DNA of HEK 293 cells by PCR using the following primers: pre-miR-122, 5'-ACTGCAGAATTGATCACATTTAC-3' (forward) and 5'-CAGTAAGTACACAAGATTGAGAAG-3' (reverse); pre-miR-34a, 5'-GCCTCCTGCATCCTTTCTTTC-3' (forward) and 5'-CCTGTGCCTTTTTCCTTCCG-3' (reverse).

\subsection{In vitro sensor evaluation}

Huh7, HeLa and HEK 293 cells were cultured in Dulbecco's modified Eagle's medium supplemented with $10 \%$ fetal bovine serum at $37^{\circ} \mathrm{C}$ in $5 \% \mathrm{CO}_{2}$. Cells were transfected with sensors using Lipofectamine 2000 (Invitrogen, Carlsbad, USA) in accord with the manufacturer's instructions. The levels of Gluc in the cell-culture supernatants were subsequently measured, to allow the functionality and sensitivity of the sensors to be evaluated.

\subsection{In vivo sensor evaluation}

Male BALB/c mice, 5-6 weeks old, were housed in pathogen-free conditions at the Institute of Laboratory Animal Science, Chinese Academy of Medical Sciences (Beijing, China). All procedures involving animals were conducted in accord with institutional guidelines, which comply with national and international regulations and guidelines. Sensors were delivered by hydrodynamic tail vein (HTV) injection as described previously [16]. Briefly, sensors were diluted in a volume of normal saline (in $\mathrm{mL}$ ) equivalent to $10 \%$ of the average body weight (in g) and were slowly injected manually into the lateral tail vein, over the course of 6-7 s. To evaluate sensor function, $2.5 \mu \mathrm{L}$ blood samples were collected at different time points by making small incisions in the tail, and Gluc activity was determined using a commercial Gaussia luciferase assay kit (New England Biolabs) in accord with the manufacturer's instructions. The number of photons produced in a $10 \mathrm{~s}$ window was counted using a luminometer (Turner BioSystems, Sunnyvale, USA).

\subsection{In vivo bioluminescent imaging}

Twenty-four hours after hydrodynamic injection of CMV/ 
A

Gluc-miRT

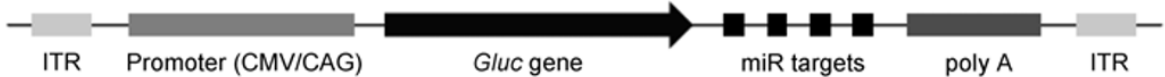

Control (Gluc)

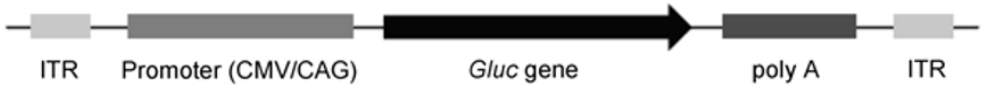

B
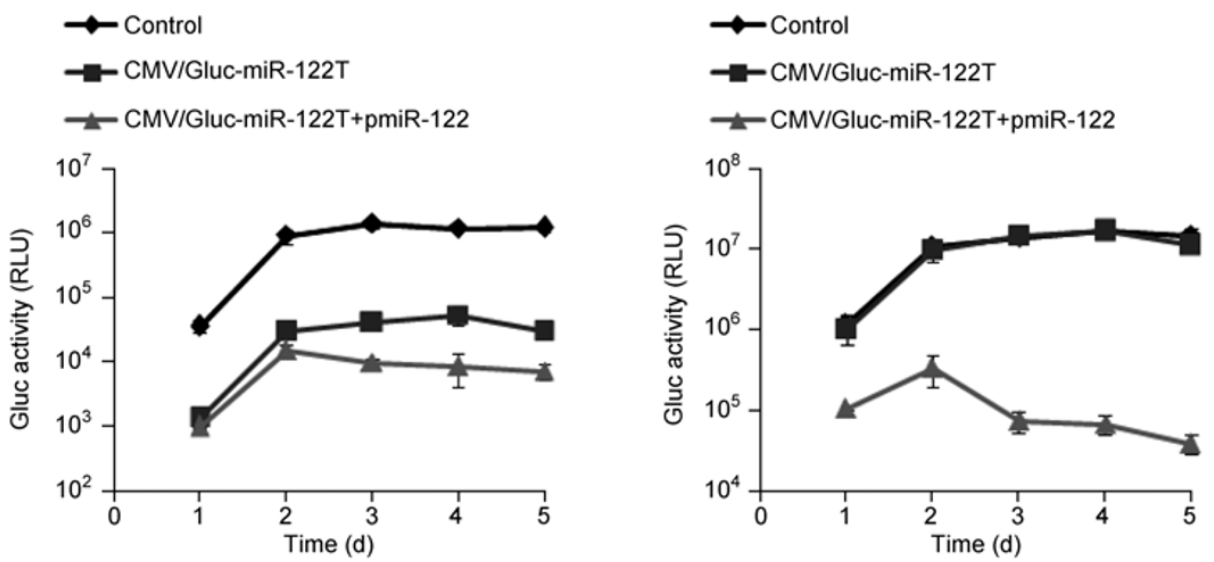

Figure 1 Gluc-miRT sensors and in vitro assessment of the CMV/Gluc-miR-122T sensor. A, The Gluc-miRT sensor was constructed by inserting four repeats of the target sequence of mature miRNA between the Gluc gene and the polyadenylation (polyA) site (upper). A Gluc expression plasmid without miRNA target sequences was constructed as a control (bottom). Gluc, Gaussia princeps luciferase; miRT, miRNA target sequences; CMV, human cytomegalovirus promoter; CAG, CMV early enhancer/chicken $\beta$-actin promoter; ITR, inverted terminal repeat from adeno-associated virus serotype 2. B, CMV/Gluc-miR-122T was transfected alone or with pmiR-122 into Huh7 cells (left) or HeLa cells (right). Gluc activity in cell culture medium was detected on days $1-5$. The results are presented as means \pm SD $(n=3)$. pmiR-122, plasmid encoding pre-miR-122; RLU, relative light units.

Fluc, $150 \mathrm{mg} \mathrm{kg}^{-1}$ body weight of D-luciferin (Molecular Imaging Products, Bend, USA) was injected intraperitoneally into mice. Ten minutes later, mice were anesthetized by intraperitoneal injection of $75 \mathrm{mg} \mathrm{kg}^{-1}$ body weight of sodium pentobarbital. Images were acquired $5 \mathrm{~min}$ after sodium pentobarbital injection. The whole-body images for luciferase activity were acquired for $10 \mathrm{~s}$ using an IVIS imaging system (Xenogen, Hopkinton, USA).

\section{5 miRNA quantification}

To eliminate any nonspecific miRNA changes, total RNA was isolated from mouse livers using a mirVana ${ }^{\mathrm{TM}}$ miRNA isolation kit (Ambion, Austin, USA). Reverse-transcription quantitative PCR was performed using a mirVana ${ }^{\mathrm{TM}}$ RT-qPCR miRNA detection kit (Ambion) in conjunction with the has-miR-122 and has-miR-142-3p primer sets (Ambion) in accord with the manufacturer's instructions. The mouse snoRNA202 primer set (Ambion) was used as a control. To determine absolute copy numbers, a standard curve was generated using HPLC-purified RNA oligonucleotides corresponding to miR-122 or miR-142-3p (Invitrogen, Shanghai, China).

\section{Results}

\subsection{In vitro assessment of the CMV/Gluc-miR-122T sensor}

The CMV/Gluc-miR-122T miRNA sensor was constructed as described in the Materials and methods section (Figure 1A). The Gluc expression plasmid without miR-122 binding sites, CMV/Gluc, was also constructed as a control (Figure 1A). To determine whether the sensor effectively measures the level of miR-122 in cells, we transfected CMV/GlucmiR-122T into Huh7 cells, which express miR-122 at high levels and into HeLa cells, which are negative for miR-122 expression [17]. Levels of Gluc secreted into the supernatant were assayed on days 1-5 after transfection. The growth medium was replaced every day to avoid Gluc accumulation. The supernatants of Huh7 cells transfected with CMV/Gluc-miR-122T showed consistently low levels of Gluc, with activities of $(1385 \pm 126),(30089 \pm 8066),(40894 \pm$ 10917), (54402 \pm 18958$)$ and $(30335 \pm 3406)$ relative light units (RLU) on days 1-5, respectively. Conversely, supernatants from control cells showed consistently high levels of Gluc activity: (35661 \pm 7469$),(874808 \pm 207580),(1355468 \pm$ 185778), (1125206 \pm 41611$),(1211542 \pm 205606)$ RLU on 
days $1-5$, respectively (Figure 1B). Gluc levels in supernatants of HeLa cells transfected with CMV/Gluc-miR$122 \mathrm{~T}$ and with control constructs were similar on days 1 and 5 (Figure 1B). To confirm that the repression of Gluc was dependent on miR-122, Huh7 and HeLa cells were co-transfected with CMV/Gluc-miR-122T and a plasmid expressing miR-122 (pmiR-122). HeLa cells transfected with CMV/Gluc-miR-122T and pmiR-122 showed marked Gluc repression on days 1-5, with Gluc activity levels of (104107 \pm 4547$),(335557 \pm 147215),(73922 \pm 22262),(67866 \pm$ 19776) and (38551 \pm 10334$)$ RLU, compared with the activity levels in cells transfected with CMV/Gluc-miR-122T alone

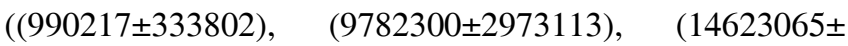
2256744), (16681655 \pm 3120897$)$ and $(11183016 \pm 2024543)$ RLU on days 1-5; Figure 1B). In Huh7 cells, co-transfection with pmiR-122 enhanced the repression of Gluc (activity $(986 \pm 64),(14933 \pm 3337),(9961 \pm 1301),(8638 \pm 4530)$ and (7192 \pm 2067$)$ RLU on days 1-5), whereas pmiR-122 further repressed Gluc activity only slightly (Figure 1B). These data suggest that Gluc was repressed by native and transduced miR-122 and that this repression was detected consistently by our CMV/Gluc-miR-122T sensor. Furthermore, our CMV/Gluc-miR-122T sensor could be used to monitor the real-time kinetics of miR-122 activity by meas- uring the levels of Gluc in the culture medium

\subsection{In vivo assessment of CMV/Gluc-miRT sensors}

We then determined whether the CMV/Gluc-miR-122T sensor could be used to monitor miR-122 levels in mouse hepatocytes which express miR-122 at very high levels [17]; miR-122 is estimated to account for $70 \%$ of the total liver miRNA content [18]. In addition to the plasmids CMV/Gluc and CMV/Gluc-miR-122T, the non-secreted Fluc expressing plasmid, CMV/Fluc, was constructed as an internal control (described in the Materials and methods section). One group of mice was inoculated with $10 \mu \mathrm{g}$ of CMV/Gluc and $10 \mu \mathrm{g}$ of CMV/Fluc by HTV injection. Another group of mice was inoculated with the same amount of CMV/ Gluc-miR-122T and CMV/Fluc. Twenty-four hours after injection, Gluc activity was detected in $2.5 \mu \mathrm{L}$ blood samples and Fluc activity was quantified using an IVIS imaging system. There was no significant difference between Fluc activity in mice co-injected with $\mathrm{CMV} /$ Fluc+CMV/Gluc $\left(\left(2.50 \times 10^{8} \pm 1.08 \times 10^{8}\right) \mathrm{RLU}\right.$; Figure $2 \mathrm{~A}$ and $\left.\mathrm{C}\right)$ or $\mathrm{CMV} /$ Fluc+CMV/Gluc-miR-122T $\left(\left(2.32 \times 10^{8} \pm 1.48 \times 10^{8}\right)\right.$ RLU; Figure $2 \mathrm{~B}$ and $\mathrm{C})$. The Gluc activity in mice co-injected

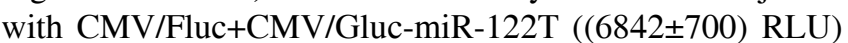

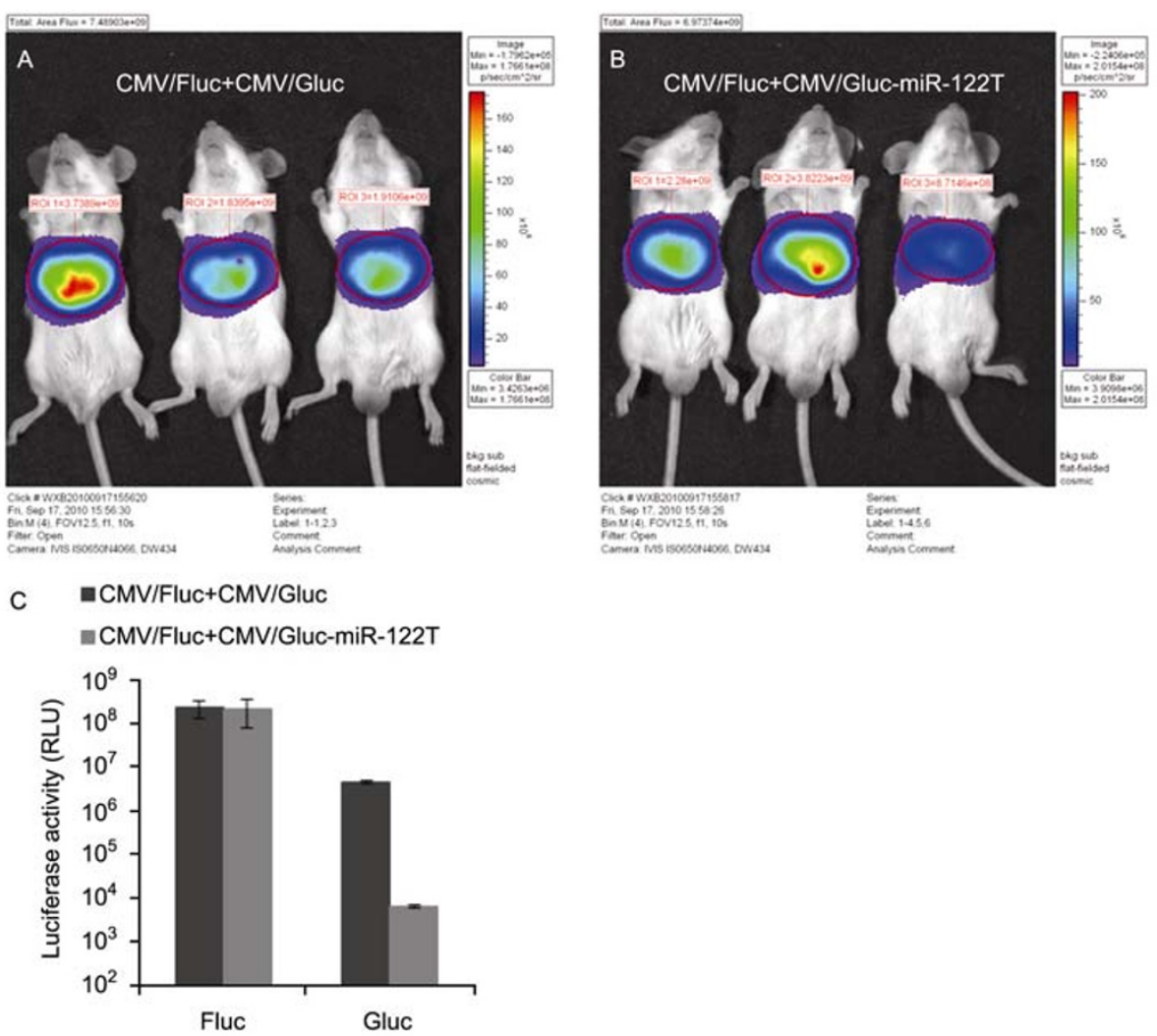

Figure 2 Detecting the activity of Gluc or Fluc in mice following co-injection of CMV/Gluc-miR-122T sensor and CMV/Fluc. A, Imaging of Fluc in mice co-injected with CMV/Fluc+CMV/Gluc. B, Imaging of Fluc in mice co-injected with CMV/Fluc+CMV/Gluc-miR-122T. C, Summary of A and B, Gluc blood assays of mice co-injected with CMV/Fluc+CMV/Gluc or mice co-injected with CMV/Fluc+CMV/Gluc-miR-122T. The results are presented as means $\pm \mathrm{SD}(n=3)$. 
showed a 696.9-fold decrease compared with mice co-injected with CMV/Fluc+CMV/Gluc ((4768176 \pm 499358$)$ RLU; Figure 2C). These data showed that Gluc of the CMV/Gluc-miR-122T sensor was significantly repressed by native miR-122 in mouse liver and that the inhibition of Gluc reflected the activity of miR-122. These results also confirmed that the change in Gluc activity was influenced by the insertion of miRNA target sites.

Sensor dose effect was then assessed. We delivered 0.1, 1,10 , or $50 \mu \mathrm{g}$ of CMV/Gluc-miR-122T sensor by HTV injection into mouse hepatocytes and Gluc was monitored continuously in $2.5-\mu \mathrm{L}$ blood samples from 2 to $96 \mathrm{~h}$ after injection. All four doses of miR-122 sensor showed similar efficiency for monitoring miR-122 in vivo (Figure 3A-D). For example, in mice injected with a $10 \mu \mathrm{g}$ dose of CMV/ Gluc-miR-122T, the Gluc activities were $(28543 \pm 5447)$, (39883 \pm 16693$),(29172 \pm 2216),(33517 \pm 10103), \quad(33047 \pm$ 9510), (18970 \pm 735$),(6625 \pm 791),(1703 \pm 116),(438 \pm 60)$ and (275 \pm 36$) \mathrm{RLU}$ at 2, 4, 6, 8, 10, 12, 24, 48, 72 and $96 \mathrm{~h}$, respectively. The corresponding Gluc activities in controls were $\quad(301251 \pm 27622), \quad(1680742 \pm 323635), \quad(4251860 \pm$ 668420), (6377790 \pm 1002630$),(9496617 \pm 1003472),(8070357 \pm$ 1366697), (4679858 \pm 706044$),(1584957 \pm 170868),(253340 \pm$ 54503) and (37228 \pm 10340$)$ RLU at 2, 4, 6, 8, 10, 12, 24, 48, 72 and $96 \mathrm{~h}$, respectively. Thus, the extent of repression was as high as 706.4-fold at $24 \mathrm{~h}$ (Figure 3C).
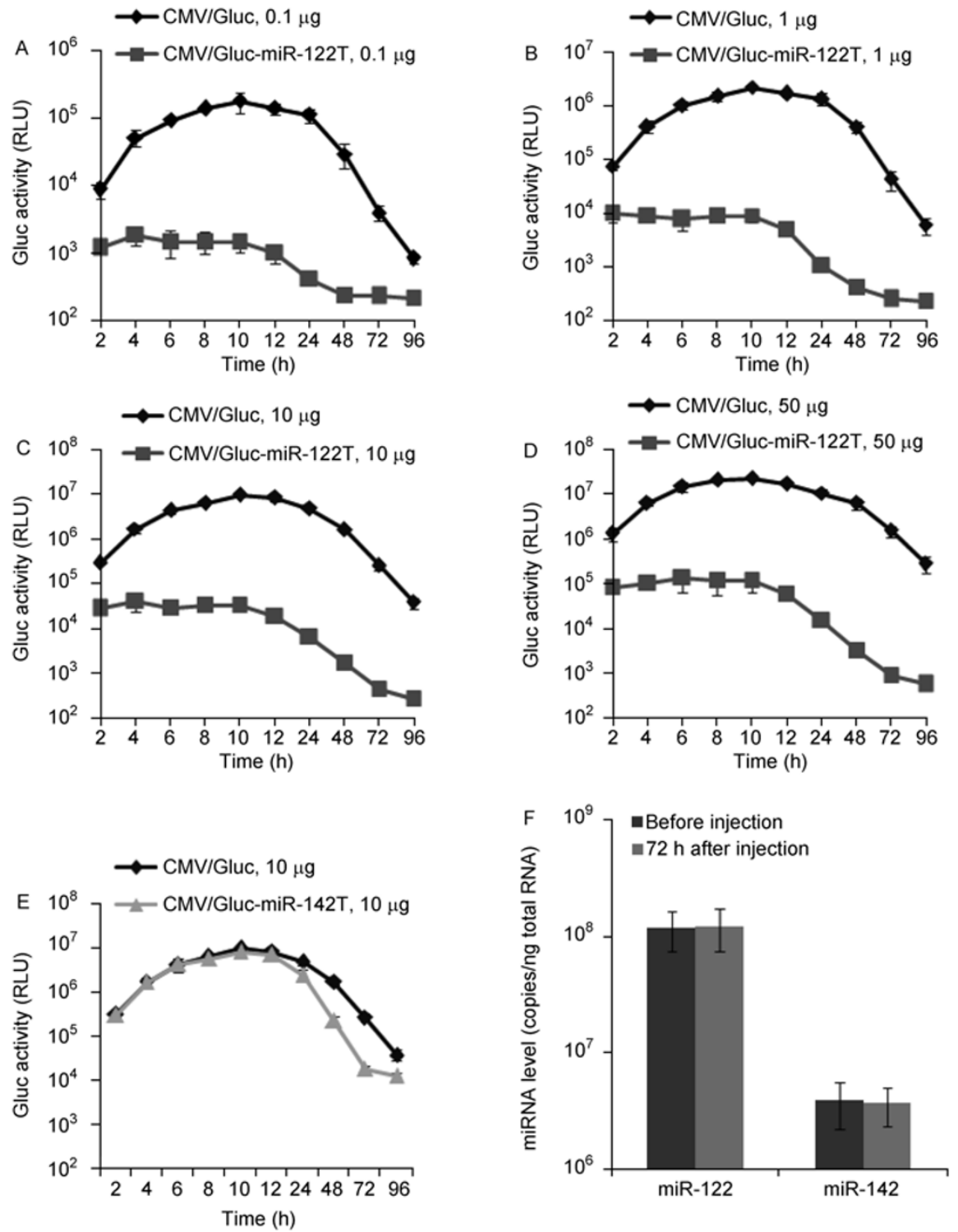

Figure 3 In vivo assessment of the CMV/Gluc-miR sensors. BALB/c mice received $0.1 \mu \mathrm{g}(\mathrm{A}), 1 \mu \mathrm{g}(\mathrm{B}), 10 \mu \mathrm{g}$ (C), and $50 \mu \mathrm{g}$ (D) of CMV/GlucmiR-122T by HTV injection and blood Gluc activity was measured. E, CMV/Gluc-miR-142T (10 $\mu \mathrm{g})$ was delivered into BALB/c mice by HTV injection, and blood Gluc activity was measured. The results are presented as means $\pm \mathrm{SD}(n=4)$. F, miRNA quantification. Total RNA was isolated from the livers of $\mathrm{BALB} / \mathrm{c}$ mice immediately before and $72 \mathrm{~h}$ after injection of CMV/Gluc-miR-122T or CMV/Gluc-miR-142T and miRNA levels were quantified by RT-qPCR using primers and probes specific for miR-122 or miR-142. The results are presented as means \pm SD $(n=4)$. 
To determine the specificity of the CMV/Gluc-miR-122T sensor, we replaced the miR-122 target sequence with that of miR-142-3p (miR-142), which is expressed at low levels in the liver [19,20]. When CMV/Gluc-miR-142T $(10 \mu \mathrm{g})$ was delivered to the mouse liver by HTV injection, no repression of Gluc activity by miR-142 was observed within $12 \mathrm{~h}$ of injection. Gluc activity subsequently declined slightly to $(2413456 \pm 635134),(211951 \pm 49479)$, (17665 \pm 2705) and (11892 \pm 2235$)$ RLU at 24, 48, 72 and $96 \mathrm{~h}$ after injection, respectively; the extent of repression was only 1.9-14.3 fold at 24-96 h (Figure 3E). Taken together, these observations indicate that our miRNA sensor reliably and conveniently detected the relevant miRNA in mouse liver.

\subsection{RT-qPCR analysis of miR-122 and miR-142 in mouse liver}

Gluc levels in mouse liver decreased after $24 \mathrm{~h}$ after injection under all test conditions. There are two possible explanations for this observation. First, HTV injection injury and the plasmids may trigger miRNA expression in the liver, thus leading to repression of the target, Gluc. Alternatively, the decrease may result from silencing of the CMV promoter in the mouse liver [21]. To examine whether HTV injection or the miRNA sensor itself affected the levels of miRNAs in the liver, we determined the levels of miR-122 and miR-142 in mouse liver by RT-qPCR, immediately before and $72 \mathrm{~h}$ after injection. The miR-122 content (copies/ng RNA) in mouse liver was $(1.20 \pm 0.46) \times 10^{8}$ and $(1.23 \pm 0.49) \times 10^{8}$ before and after injection of CMV/GlucmiR-122T, respectively. For miR-142, these values were $(3.88 \pm 1.67) \times 10^{6}$ and $(3.64 \pm 1.33) \times 10^{6}$ before and after injection of CMV/Gluc-miR-142T (Figure 3F). Consistent with previous data, these observations indicate that miR-122 is dominant in mouse liver. Moreover, the lack of change in miR-122 or miR-142 levels before and after injection indicates that HTV did not alter the expression of liver miRNAs and that the expression of miRNA binding sites in the liver did not produce obvious depressive effects on the relevant miRNAs.

\subsection{Long-term monitoring of endogenous miR-122 and miR-142 in the mouse liver using CAG/Gluc-miRT}

To determine the factors responsible for the rapid decrease in Gluc levels $24 \mathrm{~h}$ after injection and to improve our sensor, we replaced the CMV promoter in CMV/Gluc-miR-122/142T, as well as in the control, with a CAG promoter to construct CAG/Gluc-miR-122/142T and CAG/Gluc, respectively (Figure 1A). The CAG promoter has been reported to be an excellent alternative to the CMV promoter for hydrodynamic delivery of genes to the liver [22]. CMV/Gluc, CAG/Gluc, CAG/Gluc-miR-122T or CAG/Gluc-miR-142T at the dose of $10 \mu \mathrm{g}$ was delivered to mice by HTV injection and Gluc blood assays were performed. Although Gluc activity for CMV/Gluc declined enormously from $(8070357 \pm 1366697) \mathrm{RLU}$ at $12 \mathrm{~h}$ to $(687 \pm 101) \mathrm{RLU}$ at 25 days (Figure 4A), Gluc activity for CAG/Gluc remained stable for a long time, decreasing to its lowest level $((459226 \pm 53049) \mathrm{RLU})$ at day 25; this level was only 4.2-fold lower than the peak Gluc activity ((1943818 \pm 499679) RLU), recorded at $6 \mathrm{~h}$, and 2.0-fold lower than the Gluc activity at $48 \mathrm{~h}((951915 \pm 75173)$ RLU; Figure 4A). Taken together, these observations indicate that the CMV promoter was responsible for the rapid decrease in Gluc expression in the early stages of the original experiments and that the CAG promoter is an important element for long-term monitoring of miRNA in the liver. The Gluc activity of CAG/Gluc-miR-122T was depressed $3 \mathrm{~h}$ after injection and remained at a relatively stable level ranging from $(1239 \pm 381)$ RLU (highest level at $72 \mathrm{~h})$ to $(918 \pm 39)$ RLU (lowest level at day 15) from $24 \mathrm{~h}$ to 25 days post-injection (Figure 4A). The degree of repression of miR-122 was stable at 502.9- to 766.4-fold from $48 \mathrm{~h}$ after injection to day 25. For miR-142, Gluc activity was

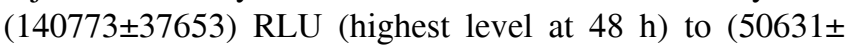
5796) RLU (lowest level at day 20) from $48 \mathrm{~h}$ after injec-
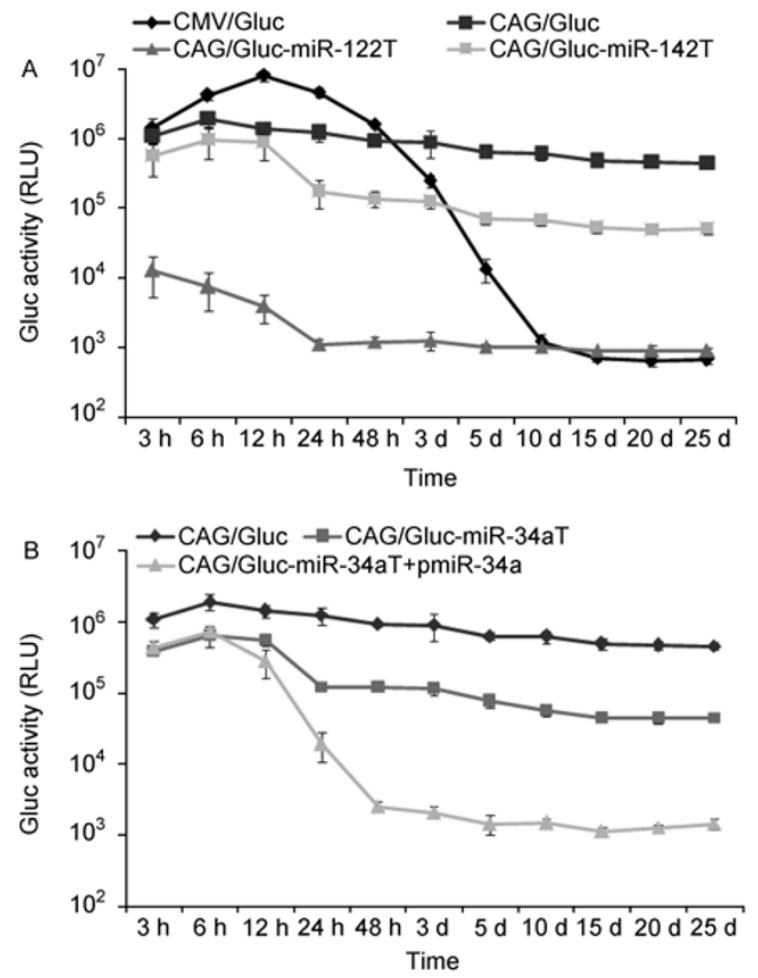

Figure 4 Long-term in vivo assessment of CAG/Gluc-miRT sensors. A, Monitoring of miR-122 and miR-142 activities in mouse livers. CMV/Gluc, CAG/Gluc, CAG/Gluc-miR-122T or CAG/Gluc-miR-142T was delivered into BALB/c mice by HTV injection, and blood Gluc activity was measured. The results are presented as means $\pm \mathrm{SD}(n=4)$. B, Monitoring miR-34a in mouse liver. CAG/Gluc-miR-34aT was delivered into BALB/c mice alone or together with pmiR-34a by HTV injection, and blood Gluc activity was measured. The results are presented as means $\pm \mathrm{SD}(n=4)$. pmiR-34a, plasmid encoding pre-miR-34a. 
tion to day 25 (Figure 4A), representing stable repression for miR-142 of 6.8- to 9.3-fold. These results indicate that compared with CMV/Gluc-miRTs, CAG/Gluc-miRTs detected miRNA levels more consistently and for a longer period. Moreover, these results demonstrate that miRNA levels were not affected by miRNA sensor injection. In conclusion, long-term expression of miRNA sensors driven by the CAG promoter allowed reliable and sensitive monitoring of miRNAs in mouse liver.

\subsection{CAG/Gluc-miRT is also suitable for monitoring changes in exogenous miRNA activity in the liver}

Having established a sensitive sensor for long-term monitoring of endogenous native miRNAs in vivo, we next evaluated the application of CAG/Gluc-miRTs to real-time monitoring of exogenous miRNAs in the liver. We performed HTV injection of $10 \mu \mathrm{g}$ of CAG/Gluc-miR-34aT (encoding the miR-34a target sequence, with miR-34a being expressed at low levels in the liver [20]) into one group of mice to detect endogenous miR-34a in the liver. In a parallel experiment, $10 \mu \mathrm{g}$ of CAG/Gluc-miR-34aT and $10 \mu \mathrm{g}$ of pmiR-34a (a plasmid encoding miR-34a) were co-transfected into another group of mice, to study the effect of miR-34a overexpression on its target. Similar to the miR-142 sensor described above, the Gluc activity from CAG/Gluc-miR-34aT was slightly repressed from $3 \mathrm{~h}$ after injection. This repression was increased at $24 \mathrm{~h}((124960 \pm$

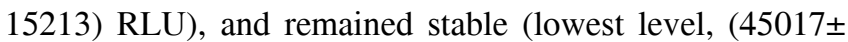
8349) RLU at day 20) until day 25 (Figure 4B). The degree of repression for CAG/Gluc-miR-34aT was 7.9- to 10.8fold from $24 \mathrm{~h}$. These results reflect the low level of native miR-34a in mouse liver. For co-transfection with pmiR-34a, slight repression, similar to that observed with CAG/GlucmiR-34aT alone, was detected at 3-6 h post-injection, but the Gluc activity decreased markedly from $12 \mathrm{~h}((284865 \pm$ 123674) RLU), falling to $(19113 \pm 8412)$ RLU at $24 \mathrm{~h}$, and then to $(2503 \pm 446)$ RLU at $48 \mathrm{~h}$, before remaining stable at a low level (lowest level, (1129 \pm 140$)$ RLU at day 15) until day 25 (Figure 4B). The degree of repression for CAG/ Gluc-miR-34aT in the pmiR-34a-co-transfection group was 319.9 - to 437.5 -fold from $48 \mathrm{~h}$ post-injection, and the greatest repression was observed 48-72 h after injection. These observations suggest that our miRNA sensors may also be useful for monitoring changes in exogenous miRNA activity in vivo.

\section{Discussion}

Since their discovery in 1993, miRNAs have been reported to have roles in development, embryogenesis, differentiation, organogenesis, growth control, programmed cell death, human diseases such as cancer and the inhibition of viral replication [1,4]. Northern blot analysis continues to be widely used as the standard miRNA detection method. Other detection techniques and tools, such as quantitative RT-PCR and microarrays, provide greater sensitivity or higher-throughput and require lower amounts of starting material [6,7]. However, these methods are time-consuming and cannot provide real-time information regarding changes in miRNA levels and functionality. Furthermore, these methods cannot be applied repeatedly to the same subject.

miRNA sensors, containing miRNA binding sites in reporter gene mRNAs, have been used to detect the presence and function of miRNAs in cells [8]. These sensors have been applied to the analysis of miRNA regulation in the liver $[23,24]$, notably for miR-122, which is specifically and abundantly expressed in the liver. For noninvasive measuring of miRNAs, optical imaging techniques using luciferase-based miRNA sensors have been used in several studies, such as in vivo imaging of miR-221 biogenesis in papillary thyroid carcinoma [10] and evaluation of miR-122 regulation in mouse liver in a single mouse at different time points [25]. Although these techniques provide a non-invasive means of continuously monitoring in vivo miRNA activity in a single animal, faster and more convenient methods are still needed.

In the present study, we established a series of novel Gluc-miRT miRNA sensors that allow convenient monitoring of miRNAs in a single animal at different time points. In in vitro studies, these sensors can be used to measure miRNA activity by detecting Gluc in cell supernatants, without the need for cell lysis; the amounts of active miRNA produced within different time periods can be measured by replacing the growth medium. In in vivo assessments, miRNA activity can be detected in as little as $2.5 \mu \mathrm{L}$ of peripheral blood.

We initially tested CMV promoter-driven Gluc sensors and found that they could detect activity of miRNAs expressed at high and low levels in the liver. By co-injection of a Fluc internal control, we confirmed that the change in Gluc expression was caused by the insertion of miRNA target sites. We then found that different doses of sensors showed similar efficiency for monitoring miRNA in vivo. RT-qPCR showed hydrodynamic injection or expression of miRNA target sequence in liver did not change the miRNA levels in the liver. Although the CMV-promoter-based sensors could be applied to the long-term monitoring of miRNAs in vitro, they were ineffective in vivo because Gluc expression decreased within $24 \mathrm{~h}$ because of CMV-promoter silencing. To generate sensors that are more suitable for use in mouse liver, we replaced the CMV promoter with the CAG promoter and demonstrated that CAG-based sensors can be used to monitor miR-122 and miR-142 activity in mouse liver over long periods. Using CAG-based sensors, we successfully detected endogenous and exogenous miR-34a activity. Thus, our sensors provide a sensitive and quantitative method for long-term ex vivo monitoring of in vivo miRNA activity. 
Our Gluc-miRT sensors have several advantages. First, the use of Gluc-miRT sensors to assay blood miRNA activity avoids the killing of test animals and allows miRNA activity to be monitored in a single animal over a long period. Second, our method is faster and more convenient than imaging assays, which involve substrate injection and anesthesia prior to measurement. Imaging assays are useful for localizing signals to specific tissues; however, a blood assay is more convenient for continuous monitoring of in vivo processes. Third, because of its short half-life in the blood (approximately $20 \mathrm{~min}$ ), Gluc does not accumulate over time, allowing dynamic miRNA activity to be monitored. Finally, Gluc-miRT sensors are extremely sensitive for monitoring miRNA activity. When we used a miRNA sensor with CMV-driven Fluc as the reporter to monitor miR-122 activity in mouse liver, repression of only 33.7-fold at $12 \mathrm{~h}$ was detectable (data not shown). Similar application of the CMV/Gluc-miR-122T sensor detected a 425.4-fold repression.

In conclusion, we have established a simple method for ex vivo monitoring of in vivo microRNAs in mouse liver. The strategy that we employed can be applied to other miRNAs, as well as to other organs and cells. The technology that we have developed has the potential to facilitate studies focused on the in vivo role of miRNAs.

This work was supported by the China Special Key Program on Infectious Diseases (Grant Nos. 2008ZX10002-023 and 2008ZX10001-012) and the Research Program from the State Key Laboratory of Molecular Virology and Genetic Engineering (Grant No. 2008-S-0003).

1 Ambros V. The functions of animal microRNAs. Nature, 2004, 431: 350-355

2 Wang Y, Kato N, Jazag A, et al. Hepatitis C virus core protein is a potent inhibitor of RNA silencing-based antiviral response. Gastroenterology, 2006, 130: 883-892

3 Carthew R W, Sontheimer E J. Origins and mechanisms of miRNAs and siRNAs. Cell, 2009, 136: 642-655

4 Kim V N. MicroRNA biogenesis: coordinated cropping and dicing. Nat Rev Mol Cell Biol, 2005, 6: 376-385

5 Válóczi A, Hornyik C, Varga N, et al. Sensitive and specific detection of microRNAs by Northern blot analysis using LNA-modified oligonucleotide probes. Nucleic Acids Res, 2004, 32: e175

6 Chen J, Lozach J, Garcia E W, et al. Highly sensitive and specific microRNA expression profiling using BeadArray technology. $\mathrm{Nu}-$ cleic Acids Res, 2008, 36: e87

7 Mestdagh P, Feys T, Bernard N, et al. High-throughput stem-loop RT-qPCR miRNA expression profiling using minute amounts of in- put RNA. Nucleic Acids Res, 2008, 36: e143

8 Mansfield J H, Harfe B D, Nissen R, et al. MicroRNA-responsive 'sensor' transgenes uncover Hox-like and other developmentally regulated patterns of vertebrate microRNA expression. Nat Genet, 2004, 36: 1079-1083

9 Brown B D, Gentner B, Cantore A, et al. Endogenous microRNA can be broadly exploited to regulate transgene expression according to tissue, lineage and differentiation state. Nat Biotechnol, 2007, 25: 1457-1467

10 Lee J Y, Kim S, Hwang do W, et al. Development of a dualluciferase reporter system for in vivo visualization of MicroRNA biogenesis and posttranscriptional regulation. J Nucl Med, 2008, 49: 285-294

11 Kim H J, Chung J K, Hwang D W, et al. In vivo imaging of miR-221 biogenesis in papillary thyroid carcinoma. Mol Imaging Biol, 2009, 11: 71-78

12 Barth S, Pfuhl T, Mamiani A, et al. Epstein-Barr virus-encoded microRNA miR-BART2 down-regulates the viral DNA polymerase BALF5. Nucleic Acids Res, 2008, 36: 666-675

13 Wurdinger T, Badr C, Pike L, et al. A secreted luciferase for ex vivo monitoring of in vivo processes. Nat Methods, 2008, 5: 171-173

14 Chung E, Yamashita H, Au P, et al. Secreted Gaussia luciferase as a biomarker for monitoring tumor progression and treatment response of systemic metastases. PLoS One, 2009, 4: e8316

15 Tannous B A. Gaussia luciferase reporter assay for monitoring biological processes in culture and in vivo. Nat Protoc, 2009, 4: 582-591

16 Liu F, Song Y, Liu D. Hydrodynamics-based transfection in animals by systemic administration of plasmid DNA. Gene Ther, 1999, 6: 1258-1266

17 Jopling C L, Yi M, Lancaster A M, et al. Modulation of hepatitis C virus RNA abundance by a liver-specific MicroRNA. Science, 2005, 309: 1577-1581

18 Lagos-Quintana M, Rauhut R, Yalcin A, et al. Identification of tissue-specific microRNAs from mouse. Curr Biol, 2002, 12: 735-739

19 Brown B D, Cantore A, Annoni A, et al. A microRNA-regulated lentiviral vector mediates stable correction of hemophilia B mice. Blood, 2007, 110: 4144-4152

20 Zhang B, Pan X. RDX induces aberrant expression of microRNAs in mouse brain and liver. Environ Health Perspect, 2009, 117: 231-240

21 Al-Dosari M, Zhang G, Knapp J E, et al. Evaluation of viral and mammalian promoters for driving transgene expression in mouse liver. Biochem Biophys Res Commun, 2006, 339: 673-678

22 Nguyen A T, Dow A C, Kupiec-Weglinski J, et al. Evaluation of gene promoters for liver expression by hydrodynamic gene transfer. Surg Res, 2008, 148: 60-66

23 Suzuki T, Sakurai F, Nakamura S, et al. miR-122a-regulated expression of a suicide gene prevents hepatotoxicity without altering antitumor effects in suicide gene therapy. Mol Ther, 2008, 16: 1719-1726

24 Wolff L J, Wolff J A, Sebestyén M G. Effect of tissue-specific promoters and microRNA recognition elements on stability of transgene expression after hydrodynamic naked plasmid DNA delivery. Hum Gene Ther, 2009, 20: 374-388

25 Cawood R, Chen H H, Carroll F, et al. Use of tissue-specific microRNA to control pathology of wild-type adenovirus without attenuation of its ability to kill cancer cells. PLoS Pathog, 2009, 5: e1000440

Open Access This article is distributed under the terms of the Creative Commons Attribution License which permits any use, distribution, and reproduction in any medium, provided the original author(s) and source are credited. 